\title{
Der Allergie-Risiko-Check - Flyer und flankierende Maßnahmen zur Information werdender Eltern
}

\author{
Sonja Floto-Stammen
}

Eingegangen: 3 December 2009/Online publiziert: 9 January 2010

(C) Birkhäuser Verlag, Basel/Switzerland 2009

Die deutsche S3 Leitlinie zur Allergieprävention wurde aktualisiert und im Frühjahr in der überarbeiteten Form verabschiedet. Dabei spielt der Bereich Ernährung eine wichtige Rolle, zumal sich grundlegende Veränderungen ergeben haben. Nachdem über lange Zeit der Tenor in Richtung „Karenz“ und Vermeidung von Kontakt mit potenten Allergenen ging, wird dies vor allem im Bereich der Ernährung, aufgrund fehlender Evidenz, nicht mehr empfohlen. Die Grundlage für die Ernährungsberatung ändert sich dadurch gravierend. Während potentielle Allergene aus Kuhmilch, Hühnerei oder Fisch in der Vergangenheit für stillende Mütter im Zweifel noch vom Speiseplan gestrichen wurden, sieht man heute durch deren Verzehr, insbesondere bei Fisch, eher vorbeugende Effekte. Zur Information von Multiplikatoren sowie werdenden Eltern über das Thema Allergieprävention, hat nun der aid infodienst den Allergie-Risiko-Check herausgebracht. Diese aktuelle und wissenschaftlich fundierte Kompaktinformation ist zusammen mit mehreren Fachgesellschaften* im

\footnotetext{
${ }^{*}$ Erstellt und unterstützt von: Präventions- und Informationsnetzwerk Allergie/Asthma e.V.; Deutscher Allergie- und Asthmabund, Deutsche Akademie für Prävention und Gesundheitsförderung im Kindes- und Jugendalter e.V.; Bundesministerium für Ernährung, Landwirtschaft und Verbraucherschutz, Gesellschaft Pädiatrische Allergologie Umweltmedizin, Aktionsplan gegen Allergien. Den Flyer „Allergie-Risiko-Check“ können Sie unter http://www.aid.de herunterladen und bis zu 100 Stück kostenfrei unter bestellung @aid.de anfordern.
}

Dr. S. Floto-Stammen ( $₫)$

aid infodienst, Verbraucherschutz, Ernährung, Landwirtschaft e.V, Heilsbachstraße 16, 53123 Bonn, Germany

e-mail: m.flothkoetter@aid-mail.de
Rahmen des Aktionsplans gegen Allergien der Bundesregierung entstanden. Sie enthält einen kurzen Fragenkatalog (Abb. 1), mit dessen Hilfe Eltern schon während der Schwangerschaft - am besten zusammen

Hat Ihr Kind ein erhöhtes Allergierisiko? Machen Sie den Test.

Welche Allergien haben Sie zurzeit oder früher gehabt? Bitte kreuzen Sie an!

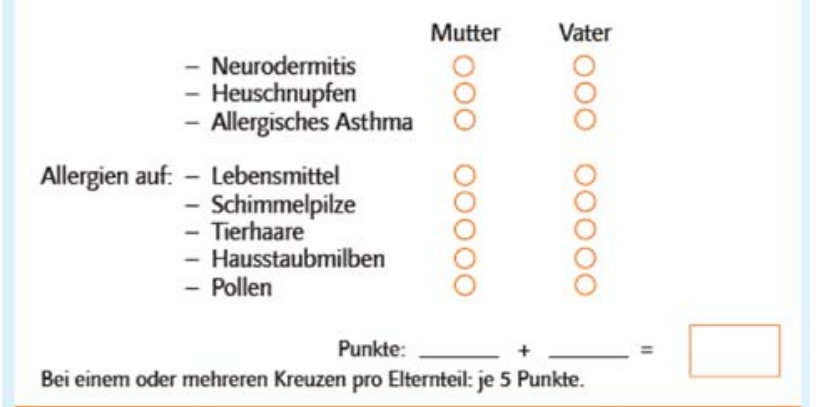

Wie viele Geschwister des Babys haben Allergien? Für jedes Kind mit Allergie: 3 Punkte.

Gibt es Katzen in Ihrem Haushalt?

Wenn Ja und bei Allergien in der Familie: 3 Punkte.

Wird in Ihrer Wohnung und/oder in Gegenwart

von Mutter oder Kind geraucht?

Wenn Ja und bei Allergien in der Familie: 5 Punkte.

Wenn ja und keine Allergien in der Familie: 1 Punkt.

Rauchen Sie als Mutter in der Schwangerschaft und/oder Stillzeit?

$\bigcirc$ Ja Nein

Wenn Ja und bei Allergien in der Familie: 5 Punkte.

Wenn Ja und keine Allergien in der Familie: 1 Punkt.

Gesamt-Punktzahl:

Abb. 1 Fragebogen des Allergie-Risiko-Check 


\section{Punkte: Es besteht kein erhöhtes Allergierisiko.}

Sie gehören zur Gruppe der Eltern, die kein erhöhtes Allergierisiko vererben und Ihrem Kind ein gesundes Umfeld bieten. Trotzdem sind die Tipps in dieser Broschüre wertvoll, um das allgemeine Allergierisiko zu mindern.

1 bis 7 Punkte: Es besteht ein erhöhtes Allergierisiko.

Mit wenigen Maßnahmen kōnnen Sie wirksam vorbeugen. Dabei gibt es grundsätzlich zwei Wege: Meiden Sie Umweltfaktoren, die Allergien fördern (z. B. Tabakrauch, Luftschadstoffe). Und unterstützen Sie Ihr Kind, vor allem durch Stillen, einen eigenen Schutz vor Allergien aufzubauen. Ab 3 Punkten: Wenn Sie Ihr allergiegefährdetes Baby nicht stillen, dann braucht es in den ersten Lebensmonaten eine allergenarme Säuglingsnahrung als Muttermilchersatz (siehe S. 22)

Ab 8 Punkten: Es besteht ein stark erhōhtes Allergierisiko.

Dies ist kein Grund zur Panik, denn vorbeugende Maßnahmen können sehr wirksam helfen. Dabei gibt es grundsätzlich zwei Wege: Meiden Sie Umweltfaktoren, die Allergien fördern (z. B. Tabakrauch, Luftschadstoffe). Und unterstützen Sie Ihr Kind, vor allem durch Stillen, einen eigenen Schutz vor Allergien aufzubauen. Wenn Sie lhr allergiegefährdetes Baby nicht stillen, dann braucht es in den ersten Lebensmonaten eine allergenarme Sāuglingsnahrung als Muttermilchersatz (siehe S. 22).

Neben der erblichen Veranlagung ist Rauchen der größte Risikofaktor für Allergien. Dabei zählt jeder Punkt, den Sie hier sammeln, als Verstärker. Der wichtigste Allergieschutz für lhr Baby ist, in seiner Gegenwart und in Räumen, in denen es sich aufhält, nicht zu rauchen. Das gilt auch bereits für die Zeit der Schwangerschaft.

Nutzen Sie alle in dieser Broschüre beschriebenen Wege zur Vorbeugung von Allergien bei Ihrem Baby. Lassen Sie sich zusätzlich von den auf Seite 46/47 genannten Allergieexperten beraten. Dann sind Sie und Ihr Baby bestens gerüstet für einen gesunden Start ins Leben.

Abb. 2 Auswertungsschema für den Fragebogen des AllergieRisiko-Check mit Arzt/Ärztin oder Hebamme - das individuelle Allergierisiko ihres Babys überprüfen können (Abb. 2). Der Flyer informiert anschließend kurz und verständlich darüber, wie Eltern in der Zeit vor und nach der Geburt das Allergierisiko ihres Kindes vermindern können. Und damit keine Frage offen bleibt, liefert er darüber hinaus Adressen geeigneter Beratungs- und Informationsangebote zu allen Themen der Allergievorbeugung wie Nichtrauchen, Stillen sowie Ernährung von Mutter und Kind. Flankierende Maßnahmen zu diesem Angebot bilden die gemeinsame persönliche Beratungshotline des Deutschen Allergie- und Asthmabundes (DAAB) und des Präventionsund Informationsnetzwerks Allergie/Asthma (PINA) sowie ein Online-Expertenforum des aid infodienst. Für die kommenden Jahre sollen darüber hinaus Fortbildungsmöglichkeiten für Multiplikatoren im Umfeld werdender Eltern zur Allergieprävention durch das Kommunikations-Netzwerk Junge Familie entwickelt und angeboten werden.

Die Änderung der Marschrichtung im Bereich Allergieprävention bedeutet eine große Herausforderung für die Information ${ }^{1}$ aller Betroffenen, sowohl im Bereich der medizinischen Betreuung als auch bei den Eltern selber.

\footnotetext{
${ }_{1}$ Telefonhotline - Allergievorbeugung: 01805/052251 Mo.- Fr. von 9:30 bis 12:00 Uhr (12 Cent/Minute). Online-Expertenforum Allergiegievorbeugung: http://www.forum-allergien-vorbeugen.de.
} 